\title{
Erythropoietin: From a Biosimilar to Innovative Products Protected by Industrial Property Assets
}

\author{
Lien López Matilla ${ }^{1}$, Pedro Bárzaga Fernández ${ }^{2}$ and Sonia González Blanco ${ }^{3}$ \\ 1. Patent Office, Centro de Inmunología Molecular, Havana 11300, Cuba \\ 2. Patent Office, Center for Pharmaceutical Research and Drug Development, Havana 10600, Cuba \\ 3. Patent Office, Center for Genetic Engineering and Biotechnology, Havana 11300, Cuba
}

\begin{abstract}
Aim: EPO (erythropoietin) is a hormone that stimulates the erythropoiesis and is mainly produced by the kidneys. In the early 1990s among the emerging biotech drugs, the recombinant human EPO (rhEPO) was the best-selling product worldwide, reaching nearly three billion dollars annually. The CIM (center of molecular immunology) produced and sold the rhEPO as commercial strategy to recover the investment made in its new facilities. This work summarizes the inventions that protect the innovative products developed by three Cuban institutions, starting from rhEPO, and the industrial property strategy followed by them. Methods: The information was obtained from the United States Patent, Trademark Office (USPTO) database, Patentscope, Espacenet, patent databases of Center for Pharmaceutical Research and Drug Development (CIDEM) and Cuban Industrial Property Office. Conclusions: The manufacturing process of CIM's EPO has its own patent family. From a manufacturing by product an innovative formulation protected by patent was obtained. There is a patent family around the nasal formulation and it continues enlarging. From a biosimilar pharmaceutical innovative products impacting on human health have been obtained.
\end{abstract}

Key words: Erythropoietin, ataxia, pegylated EPO, biosimilar.

\section{Introduction}

Since the beginning of XX century many studies contributed to the conception of a circulating erytroid-stimulating hormone. In 1906, Paul Carnot and Clotilde Deflandre, proposed the idea that hormones regulate the production of red blood cells,after conducting experiments on rabbits subject to bloodletting. Further studies by K. R. Reissman and Allan J. Erslev demonstrated that a certain substance, circulated in the blood, is able to stimulate red blood cell production and increase hematocrit [1]. This substance was finally purified by Golwasser and Kung with over 95\% purity and coined as EPO (erythropoietin) opening doors for therapeutic uses in diseases such as anemia [2].

EPO is a $30.4 \mathrm{kDa}$ glycoprotein of 165 amino acids [3]. EPO is naturally produced by interstitial fibroblasts

Corresponding author: Lien López Matilla, M.D. research fields: immunology. in the kidney and in perisinusoidal cells in the liver. While liver production prevails in the fetal and perinatal period, renal production is predominant during adulthood. EPO stimulates red blood cell production in the bone marrow and also it plays an important role in the brain's response to neuronal injury [4].

EPO is highly glycosylated, and its carbohydrate portion contributes $40 \%$ of its molecular weight. This glycosylated moiety exhibits several glycosylation patterns, determining the existence of different isoforms. It contains up to 14 sialic acid residues. As the variable nature of the sialic acid content gives rise to EPO isoforms with differences in charge [5], they can be indistinctly detected by electrophoresis or isoelectric focusing followed by immunoblotting [6] The N-linked polysaccharideside chains appear to be important for the biosynthesis and secretion of EPO, enhancing its stability in blood, and limitinghepatic clearance, thus facilitating the systemic transit of EPO 
from kidney to bone marrow $[7,8]$.

The EPO's gene sequence and the procedure to obtain recombinant human EPO (rhEPO) were claimed in US patents 4,703,008 [9] and 5,441,868 [10], respectively. rhEPO is commercially available since 1980s, after FDA approval, it is widely employed in patients with kidney failure who undergo hemodialysis and to counteract the side effects of chemotherapy in oncology patients. In the early 1990s, rhEPO from several brands, indicated for anemia, were among the top selling products, with sales of near three billion dollars annually, reaching the blockbuster status. At that time, Cuba only imported small amounts of rhEPO, due to the high cost of each bulb, and very few patients were benefited [11]. In said situation, as strategic issue in its commercial policy, the CIM decided to undertake the manufacture of rhEPO and to sale it. This allowed full coverage of domestic demand of patients with anemia, due to renal failure, and the recovery of the investment made in its new manufacturing facilities [12].

This work summarizes the inventions that protect the innovative products developed by three Cuban institutions, starting from rhEPO, and the industrial property strategy followed by them.

\section{Method and Materials}

\subsection{Patent around the Methods of Obtaining of rhEPO}

In order to find patents around rhEPO, the information was obtained from United States Patent and Trademark Office (USPTO) database (https://www.uspto.gov/patents-application-process/se sear-patents). After that, a freedom to operate study was performed to determine whether the manufacture of rhEPO in Cuba could be done without infringing valid industrial property rights of the rhEPO's patents owners. For said purpose, the information was gathered from the web site of the Cuban Industrial Property Office (http://www.ocpi.cu).

\subsection{Patents around Basic Form of EPO}

A study of the state of the art around basic forms of
EPO5 was performed by consulting Patentscope (http://patentscope.wipo.int) and Espacenet (http://www.epo.org/searching/free/espacenet) databases. The information about patent on basic forms of rhEPO was obtained from CIDEM patent database.

\subsection{Patent on Pegylated rhEPO}

The study of the state of the art about pegylated rhEPO was performed by consulting Patentscope (http://patentscope.wipo.int), Espacenet (http://www.epo.org/searching/free/espacenet) and USPTO (https://www.uspto.gov/patents-applicationprocess/search-patents) databases.

\section{Results and Discussion}

\subsection{Patent around Themethods of Obtaining rhEPO}

Although in the 90's the US patent 5,441,868 was still in force, there was no corresponding patent in Cuba. To cover $100 \%$ of the Cuban patients that suffered anemia due to renal failure at that time, CIM developed its own process for fermentation and purification of rhEPO starting from CHO cells. It is said that the process was claimed in the Cuban patent No.CU 22709. At present, the rhEPO produced by CIM is commercialized under the trademark ior EPOCIM ${ }^{\circledR}$ and it has 61 sanitary approvals in different indications such as: anemia treatment in patients with chronic renal disease who undergo dialysis or pre-dialysis; anemia treatment in AIDS patients undergoing a regime with Zidovudine; cancer patients with chemotherapy induced anemia andprophylaxis and treatment of anemia in preterm newborn. ior EPOCIM ${ }^{\circledR}$ is a biosimilar product that allowed the full coverage of Cuban patients with anemia, due to renal dysfunction. Biosimilars are proteins that are similar to innovator biologics and show no clinically significant differences with the innovator medicine. They are comparatively cheaper than originator biologics, due to the shorter process for approval. Due to said reasons, they offer more alternatives to the physicians, and provide more treatment options to their patients [13]. 


\subsection{Patents around Basic Form of EPO}

The researchers of CIDEMin Havana studied the properties of the rhEPO with low content of sialic acid. Said fraction was routinely discarded in the purification process of CIM's rhEPO. This fraction has less than 9 sialic acid moieties per protein molecule, is not biologically active when is administered by systemic route, due to its extremely short plasma half-life. It was named Neuro-EPO. The researchers of CIDEM surprisingly found that intranasal administration of rhEPO with low content of sialic acid (Neuro-EPO) have a therapeutic effect on nervous cells. The above result has an added value because it represents a great economic advantage, allowing employing a fraction that was previously considered a waste in the production of rhEPO.

A new nasal formulation having as active principle the rhEPO with low content of sialic acid was developed and tested in an ischemia model in Mongolia gerbils. Three groups of animals were employed in order to evaluate the Neuro-EPO: a healthy control, a non-treated ischemic animals and ischemic animals treated with Neuro-EPO. The neurological status and motor-functional exploratory activity of each animal was determined and appeared depressed in non-treated ischemic animals, while this activity remained similar to the healthy controls in the animals treated with the nasal formulation. Also, the erythropoietic response was explored and no significant erythropoietic activity was observed, which was an important aspect, because this kind of formulations that reach the central nervous system must avoid stroke. The above mentioned results supported a new patent application filed at the Cuban Patent Office, claiming said nasal formulation and its use in neurological diseases, in general. The international application was published as WO 2007/009404. Figs. 1 and 2 (corresponding to Figs. 3 and 4 of said patent application) show the comparison in terms of motor-functional exploratory activity and neurological status, respectively, among different groups of experimental animals in the ischemia model.

Lacking biomanufacturing capabilities, CIDEM licensed the invention on Neuro-EPOto CIM for production and marketing of said pharmaceutical composition that at present is under development.

SCAs (Spinocerebellar ataxias) belong to a group of autosomal-dominant cerebellar ataxias that are classified according to specific genetic mutations. SCA type 2 (SCA2) is a polyglutamine disease caused by expansion of CAG triplet repetitions in the coding region of the ATXN2 gene [14]. SCA2 is the second most common autosomal dominant cerebellar ataxia

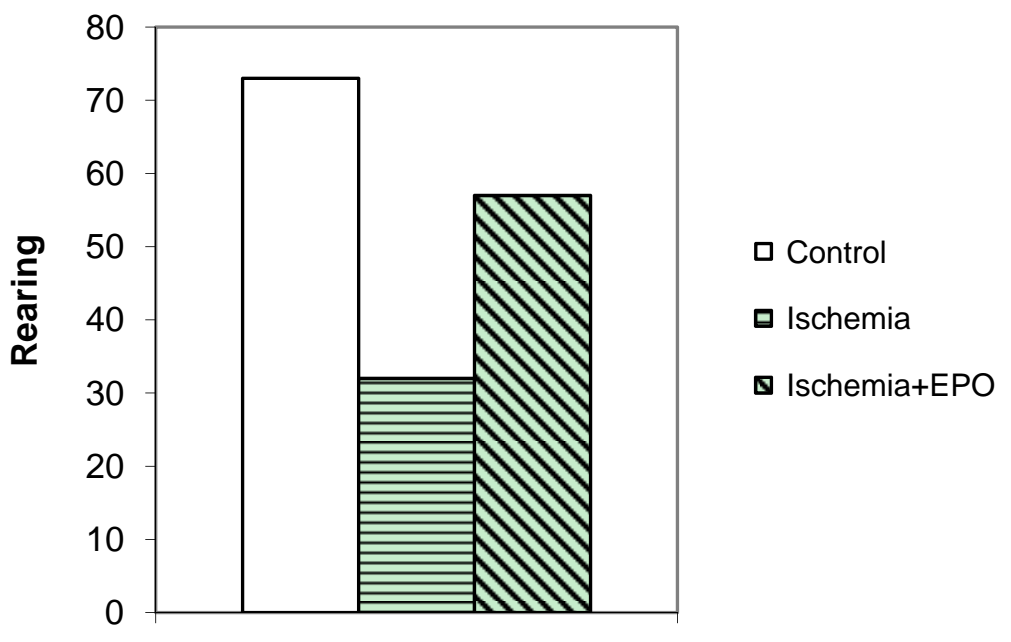

Fig. 1 Comparison of motor-functional activity observed in the ischemic model in Mongolia gerbils. 


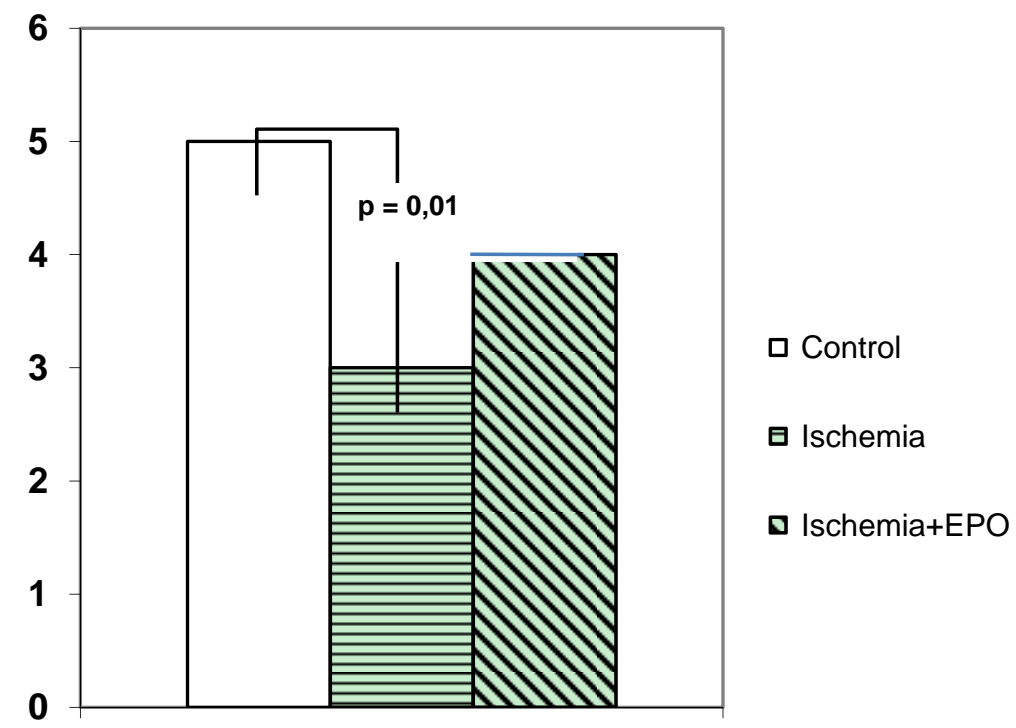

Fig. 2 Neurological status of the animals in the ischemic model, 24 hours after the injury.

worldwide [15] with the highest prevalence rates in Holguín, Cuba, where the prevalence ate of mutation carriers approaches 180 per 100,000 inhabitants [16].

Taking into account the properties of the developed nasal formulation that contains rhEPO with low sialic acid content (Neuro-EPO), the effect of such formulation was explored in a group of ataxic mice, compared to a group of ataxic mice treated with placebo. In addition, a control group of healthy untreated animals was employed. The neurological behavior of the animals treated with the rhEPO nasal formulation was similar to one observed in the healthy controls, while the animals who received placebo showed a reduced mobility. Also, as times goes by the differences, in terms of survival, between the group of untreated ataxic animals and the treated animals significantly increased, as it is shown in Fig. 3.

The above mentioned preclinical data allowed continuing to a phase I-IIa clinical trial, in patients with a clinical diagnosis of SCA2 in early stages (I-II) of the disease. Patients were divided into two groups, one received the rhEPO nasal formulation and the other group received placebo. A significant clinical progress associated with improvement of motor symptoms was observed in the group of patients treated with the
rhEPO with low sialic acid content, while the group of patients who received placebo did not show such a benefit. Said results evidenced the influence of the referred treatment on the most significant parameters that characterize the SCA2 disease and consequently on the deceleration of the disease progression.

The surprising results obtained when the nasal formulation having as active principle the rhEPO with low content of sialic acid (Neuro-EPO) was employed in the treatment of SCA2, supported the filing by CIM of a patent application claiming Cuban priority, with the application No.CU-2016-0092. The international PCT filing was done in June 2017 (PCT/CU2017/050005). The effect of Neuro-EPO on nervous cells was also published in Parra and Rodríguez 2012 [17], Maurice et al, 2013 [18] and Rodríguez et al, 2017 [19]. New clinical trials to test the efficacy of the basic rhEPO in other indications are designed and will be conducted, so new patent applications could arise.

\subsection{Patent on Pegylated rhEPO}

Biobetters are a kind of products containing the same active pharmaceutical ingredient as the marketed product, but have some molecular modifications that 


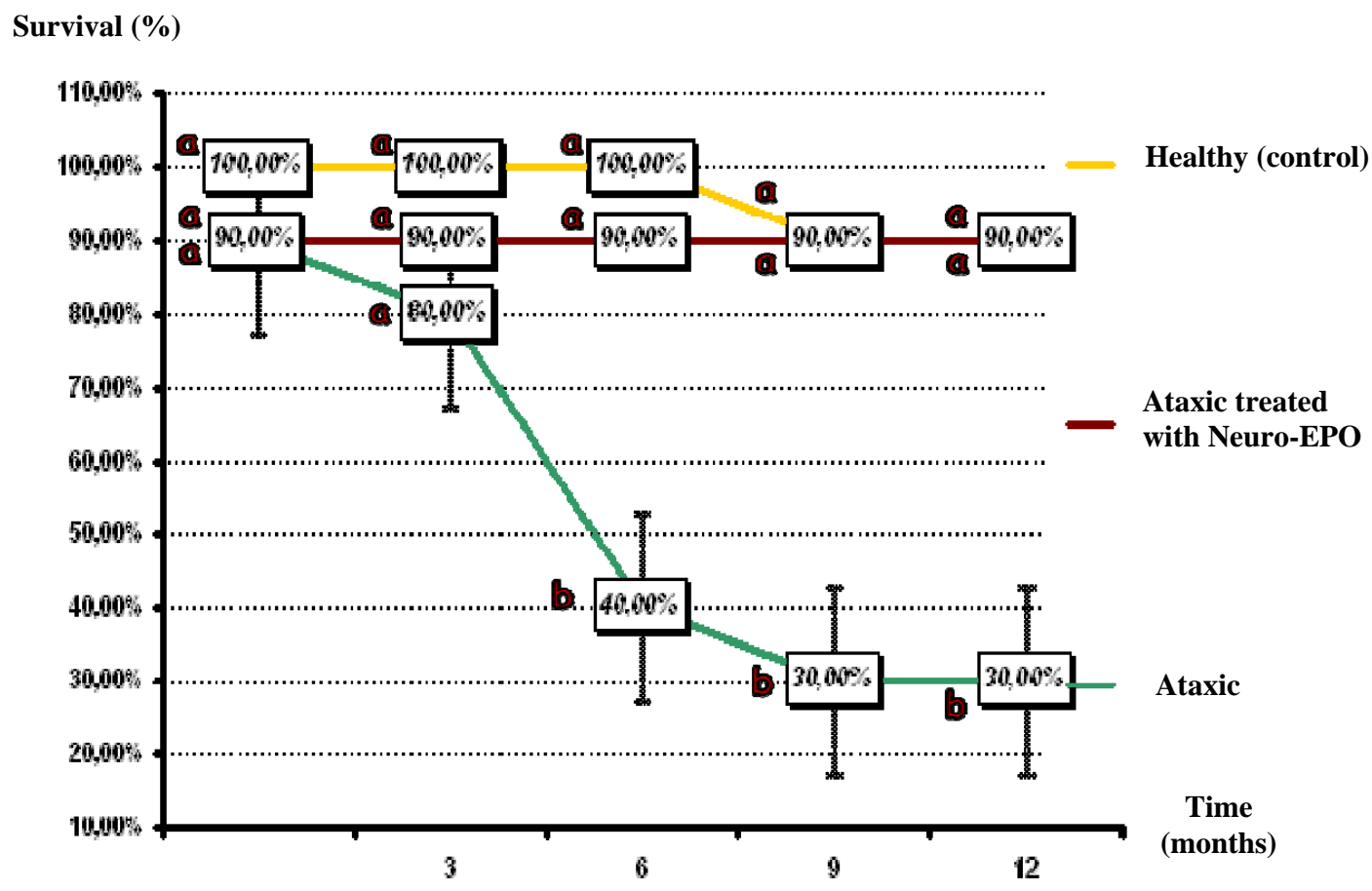

Fig. 3 Comparison of the survival in untreated ataxic animals and the animals treated with Neuro-EPO.

cause an improvement in one or more attributes over the original one. These products may arise as a strategy to extend the monopoly into the market beyond the patent expiration date of the original product. Biobetters can arise from chemical modifications such as pegylation and glycosylation; new formulations like those for controlled release; and those intended to be delivered by new administration routes. Changes in the manufacturing process, such as the use of a new cell platform, can also end in the development of a biobetter [20].

rhEPO must be administered relatively frequently, generally 3 times a week by parecteral route due to its short half-life. Consequently, it is difficult to administer said hormone for patients who need therapy of long duration. With the objective of increasing the half-life of rhEPO, several strategies have been used. For example, mutations were carried out within the molecule to increase the sites of $N$-glycosylation [21], and the protein was incorporated in microspheres [22] orliposomes [23]. Another strategy followed to increase the half-life of rhEPO was pegylation.
Mircera ${ }^{\circledR}$ is an example of a biobetter and it is the reference product for pegylated rhEPO. This product is protected by US Patent No. 7,202,208 [24].

Following the same approach, the researchers of Center for Genetic Engineering and Biotechnology (CIGB) and CIM obtained a monofunctional branched polyethylene-glycol (PEG) structure, with a molecular size similar to that claimed in US Patent No. 7,202,208, but with two PEG strands of different molecular masses. Said asymmetric PEG moleculewas conjugated to rhEPO. This alternative, unexpectedly, confers new advantages to the drug that contains it as an active pharmaceutical ingredient.

The use of an asymmetric branched PEG compound, instead of the linear one claimed in US Patent No. $7,202,208$, allowed to reduce the formation of bipegylated contaminants, and unexpectedly increased the half-life of the biomolecule, and its physicochemical stability. In addition, the biological activity of the pegylated molecule was similar to that of the unmodified rhEPO, despite that the loss of biological activity of biomolecules, due to the pegylation, 
<smiles>CCCCC(NC(=O)OCCC)C(=O)O</smiles>

Fig. 4 Schematic representation of the asymmetric branched PEG compound mentioned in US Patent Application No. 2016/0317674.

has been widely reported in the literature [25]. For the above reasons, in a patent family that includes the US Patent Application No. 2016/0317674, a conjugate comprising rhEPO and an asymmetric branched polymeric structure, comprising two branches of PEG, is claimed. The asymmetric branched PEG compound is represented in a schematic representation in Fig. 4. This application is entitled by CIGB and CIM.

\section{Conclusions}

The manufacturing process of the rhEPO produced by CIM could be protected in the Cuban Patent No. CU 22709. The rhEPO with low content of sialic acid isoform (Neuro-EPO) discarded in the established process was included in a nasal formulation, showing unexpected results that were claimed in the International Application No. WO2007/009404. In addition, through the pegylation of the CIM's rhEPO with an asymmetric branched polymeric structure, a biobetter product was obtained and it was claimed in the US Patent Application No. 2016/0317674. The strategy followed by CIM with the rhEPOhas been successful, allowing obtaining innovative products starting from a biosimilar. Around CIM's rhEPO there is a family of patents and work to enlarge it is in progress. Fortunately, all these products have an impact on human health. The collaboration between the biotech companies involved in obtaining the results showed in this paper allowed to find complementary technologies, extending the patent protection of their products, and bolstering their product portfolio.

\section{References}

[1] Bunn, H. F. 2013. “Erythropoietin.” Perspect Med 3 (3): 1-20.

[2] Jelkmann, W. 2007. "Erythropoietin After a Century of Research: Younger than Ever.” Eur J Haematol 78 (3): 183-205.

[3] Lanfranco, F., and Strasburger, C. J. 2016. "Frontiers of Hormone Research.” Sports Endocrinology 47: 115-27.

[4] Sirén, L., Fratelli, M., Brines, M., Goemans, C., Casagrande, S., Lewczuk, P., Keenan, S., Gleiter, C., Pasquali, C., Capobianco, A., Mennini, T., Heumann, R., Cerami, A., Ehrenreich, H., and Ghezzi, P. 2001. "Erythropoietin Prevents Neuronal Apoptosis after Cerebral Ischemia and Metabolic Stress.” Proc Natl Acad Sci USA 98 (7): 4044-9.

[5] Weidemann, A., and Johnson, R. S. 2009. "Non-renal Regulation of EPO Synthesis.” Kidney Int. 75: 682-8.

[6] Mocini, D., Leone, T., Tubaro, M., Santini, M., and Penco, M. 2007. "Structure, Production and Function of Erythropoietin: Implications for Therapeutical Use in Cardiovascular Disease." Curr Med Chem. 14: 2278-87.

[7] Boissel, J. P., Lee, W. R., Presnell, S. R., Cohen, F. E., and Bunn, H. F. 1993. "Erythropoietin Structure-Function Relationships: Mutant Proteins that Test a Model of Tertiary Structure.” J Biol Chem. 268: 15983-93.

[8] Obeagu, E. I. 2015. “A Review on Erythropoietin.” Int. J. Adv. Res. Biol. Sci. 2 (4): 35-47.

[9] Fu-Khen, L. 1987. DNA Sequences Encoding Erythropoietin. US Patent 4,703,008, filed November 30, 1984 and issued October 27, 1987.

[10] Fu-Khen, L. Production of Recombinant Erythropoietin. US Patent 5,441,868, filed October 23, 1987 and issued August 15, 1995.

[11] Lage, A. 2007. “Knowledge Economy.” Center of Molecular Immunology, Havana, Cuba.

[12] Cardoso, A. 2014. "Evaluation of the Cellufine PB Chromatographic Matrix in the Purification Process of hrEPO.” M.Sc. thesis, Faculty of Chemical Engineering, CUJAE, Havana, Cuba.

[13] Ravi, R. A., Bhagya, M. S., and Purushottam, S. K. 2014. "Biologics and Biosimilars: Role in Modern Pharmacotherapy and Importance of Pharmacovigilance.” Int J Res Med Sci. 2 (2): 382-6.

[14] Velázquez, L., Rodríguez, R., García, J. C., Almaguer, L. E., Cruz, T., and Laffita, J. M. 2011. "A Comprehensive Review of Spinocelebellar Ataxia Type 2 in Cuba." Cerebellum 10: 184-98.

[15] Schöls, L., Bauer, P., Schmidt, T., Schulte, T., and Riess, O. 2004. “Autosomal Dominant Cerebellar Ataxias: 
Clinical Features, Genetics, and Pathogenesis.” Lancet 3: 291-304.

[16] Rodríguez, R., Velázquez, L., Aguilera, R., Seifried, C., Peña, A., Canales, N., Medrano, J., Estupiñan, A., Vázquez, Y., González, Y., and Laffita, J. M. 2014. "Executive Deficit in Spinocerebellar Ataxia Type 2 Is Related to Expanded CAG Repeats: Evidence from Anti-saccadic Eye Movements.” Brain and Cognition 91: 28-34.

[17] Parra, A. L., and Rodríguez, J. C. 2012. "Nasal Neuro EPO Could Be a Reliable Choice for Neuroprotective Stroke Treatment." Cent Nerv Syst Agents Med Chem 12 (1): 60-8.

[18] Maurice, T., Mustafa, M. H., Desrumaux, C., Keller, E., Naert, G., de la, C., García Barceló, M., Rodríguez, Y., and García, J. C. 2013. "Intranasal Formulation of Erythropoietin (EPO) Showed Potent Protective Antivity against Amyloid Toxicity in the A $\beta 25-35$ Non-transgenic Mouse Model of Alzheimer Disease.” J Psychopharmacol 27 (11): 1044-57.

[19] Rodríguez, Y., Strehaiano, M., Rodríguez, T., and Maurice, T. 2017. "An Intranasal Formulation of Erythropoietin (Neuro-EPO) Prevents Memory Deficits and Amyloid Toxicity in the APPSwe Transgenic Mouse
Model of Alzheimer's Disease”. J Alzheimer Dis 55 (1): 231-48.

[20] Malkin, B. 2012. Biobetters and Extending into the Marketplace beyond Patent Expiration. FDA Lowyers Blog. http://www.fdalawyersblog.com. Accessed on 14 November, 2016.

[21] Burke, P. 2002. "Device for the Sustained Release of Aggregation-stabilized, Biologically Active Agent.” US Patent Application No. 2003/0133979 Filed October 22.

[22] Morlock, M., Kissel, T., Li, Y. X., Koll, H., and Winter, G. 1998. "Erythropoietin Loaded Microspheres Prepared from Biodegradable LPLG-PEO-LPLG Triblock Copolymers: Protein Stabilization and In vitro Release Properties.” J Control Release 56 (1-3): 105-15.

[23] Moriya, H., Maitani, Y., Shimoda, N., Takayama, K., and Nagai, T. 1997. "Pharmacokinetic and Pharmacological Profiles of Free and Liposomal Recombinant Human Erythropoietin after Intravenous and Subcutaneous Administrations in Rats.” Pharm Res 14 (11): 1621-28.

[24] Papadimitriu, A. 2004. Erythropoietin Compositions. US Patent 7,202,208, filed February 17, 2004 and issued April 10, 2007.

[25] Harris, J. M., and Chess, R. B. 2003. "Effect of Pegylation on Pharmaceuticals.” Nat Rev Drug Discov 2: 214-21. 